\title{
HYPERFINITENESS AND THE HALMOS-ROHLIN THEOREM FOR NONSINGULAR ABELIAN ACTIONS ${ }^{1}$
}

\author{
J. FELDMAN AND D. A. LIND
}

\begin{abstract}
THEOREM 1. Let the countable abelian group $G$ act nonsingularly and aperiodically on Lebesgue space $(X, \mu)$. Then for each finite subset $A \subset G$ and $\varepsilon>0 \exists$ finite $B \subset G$ and $F \subset X$ with $\{b F: b \in B\}$ disjoint and $\mu\left[\left(\bigcap_{a \in A} B-a\right) F\right]>1-\varepsilon$.

THEOREM 2. Every nonsingular action of a countable abelian group on $a$ Lebesgue space is hyperfinite.
\end{abstract}

1. Introduction. The principal results here are a Halmos-Rohlin theorem for nonsingular actions of a countable abelian group on a Lebesgue measure space, and a proof of their hyperfiniteness. The latter fact has relevance for the group-measure space construction of von Neumann algebras. This construction produces algebras of type III precisely when there is no equivalent measure preserved by the action (see [9, Chapter 4.2]).

These results have already been proved for measure-preserving actions. Katznelson and Weiss [4] and Conze [1] proved a Halmos-Rohlin theorem for measure-preserving actions of $\mathbf{Z}^{d}$, and Krieger [5] extended this to countable abelian groups. Hyperfiniteness was shown in the measure-preserving case by Dye in the second of his pioneering papers [2] and [3]. However, it seems worthwhile to give a simpler proof of hyperfiniteness even in this case. Finally, Veršik [10] has announced a proof of the hyperfiniteness of nonsingular countable abelian actions. However, the oniy proof of his of which we are aware [11] has serious gaps.

After this paper was completed, we learned from A. Connes that he and W. Krieger have also proved Theorem 2, apparently by somewhat different methods.

2. The Halmos-Rohlin theorem. All transformations act on a fixed Lebesgue measure space $(X, \mu)$ (see [8] for the properties of such spaces). An invertible measurable transformation of $X$ is called nonsingular if both it and its inverse map $\mu$-null sets to $\mu$-null sets. The group of all such transformations is denoted by $\mathcal{X}(\mu)$.

Let $G$ be a countable abelian group. A nonsingular action of $G$ on $(X, \mu)$ is a homomorphism $T: G \rightarrow$ N $(\mu)$. We abbreviate $T(g)(x)$ by $g x, T(g)(F)$ by

\footnotetext{
Received by the editors March 1, 1975.

AMS (MOS) subject classifications (1970). Primary 28 A65.

Key words and phrases. Group action, hyperfinite.

1 This work was partially supported by NSF Grant GP 15735 and the Miller Institute for Fundamental Research.
} 
$g F$ for subsets $F$ of $X$, etc. We say $G$ acts aperiodically on $(X, \mu)$ if the only element of $G$ which has a fixed point is the identity of $G$. If $B \subset G$ and $F \subset X$, then $B F$ denotes $\cap\{b F: b \in B\}$. A subset $F$ of $X$ is called a $B$ set if $\{b F: b \in B\}$ is a disjoint collection. If $A$ and $B$ are subsets of $G$, then $\cap_{A} B$ denotes $\cap\{B-a: a \in A\}$. We are now prepared to state the result of this section.

THEOREM 1. Let the countable abelian group $G$ act nonsingularly and aperiodically on the Lebesgue space $(X, \mu)$. Then for each finite subset $A$ of $G$ and each $\varepsilon>0$, there exists a finite subset $B$ of $G$ and a measurable $B$ set $F$ with $\mu\left[\left(\cap_{A} B\right) F\right]>1-\varepsilon$.

Proof. The proof builds on some ideas in [7]. For measure-preserving actions this proof is actually quite simple. Most of our proof is concerned with using averaging arguments to control the size of sets which are easily shown to be small for measure-preserving actions.

First, suppose that the theorem is true for actions of the $d$-dimensional integers $Z^{d}$. If $G$ is a countable abelian group acting on $X$, and $A$ is a finite subset of $G$, we can assume without loss that $A$ generates $G$. Hence for some integer $d$ and finite group $H$, we have $G$ isomorphic to $\mathbf{Z}^{d} \oplus H$. Let $\zeta$ be the partition of $X$ into orbits of $H$, and $q: X \rightarrow X / \zeta$ be the quotient map. Then $\zeta$ is a measurable partition in the sense of Rohlin [8], and $X / \zeta$ is a Lebesgue space under the measure $\mu_{\zeta}(E)=\mu\left(q^{-1} E\right)$. The aperiodic action of $\mathbf{Z}^{d}$ on $X$ induces one on $X / \zeta$. Let $A_{0}$ be the projection of $A$ into $\mathbf{Z}^{d}$. By our initial assumption, for every $\varepsilon>0$ there is a $B_{0} \subset \mathbf{Z}^{d}$ and $F_{0} \subset X / \zeta$ with $\mu_{\zeta}\left[\left(\cap_{A_{0}} B_{0}\right) F_{0}\right]>1-\varepsilon$. Let $F \subset X$ be a measurable cross section of $q$ restricted to $q^{-1}\left(F_{0}\right)$, and $B=B_{0} \oplus H$. Then $F$ is a $B$ set, and since $\cap_{A} B=\left(\cap_{A_{0}} B_{0}\right) \oplus H$, we have

$$
\mu\left[\left(\bigcap_{A} B\right) F\right]=\mu_{\xi}\left[\left(\bigcap_{A_{0}} B_{0}\right) F_{0}\right]>1-\varepsilon .
$$

Thus we may assume that $G=\mathbf{Z}^{d}$. The basic strategy is the following. We begin by showing that for arbitrarily large cubes $Q$ in $\mathbf{Z}^{d}$ there is a $Q$ set $F$ such that for every $t$ in a cube one fourth the size of $Q$, the set $(Q+t) F$ fills out at least a fixed proportion of $F$. The same argument is applied to fill out a fixed proportion of the remainder of $X$ with a smaller cube, and these two constructions are combined to fill out a larger proportion of the entire space using the smaller cube. This combination involves an averaging argument which uses some flexibility in the original choice of $Q$. Repeating this procedure eventually fills out as much of $X$ as desired.

Let $Q_{P}$ be the cube $\{0,1, \ldots, P-1\}^{d}$, and

$$
R_{P}=\{-P,-P+1, \ldots, P-1\}^{d},
$$

so that $R_{P}$ is made up of $2^{d}$ translates of $Q_{P}$. Let $B_{N}\left(Q_{P}\right)$ be a barrier of thickness $N$ surrounding $Q_{P}$, namely

$$
B_{N}\left(Q_{P}\right)=\left\{t \in \mathbf{Z}^{d}:-N \leqslant t_{j}<P+N\right\} \backslash Q_{P} .
$$

If $L$ divides $P$, let $S_{L}\left(Q_{P}\right)$ be $Q_{P}$ "shrunk" symmetrically so that a proportion 
$1 / L$ is removed from its surface, namely $S_{L}\left(Q_{P}\right)=\left\{t \in \mathbf{Z}^{d}: P / L \leqslant t_{j}\right.$ $<P-P / L\}$.

Say an integer $L$ works for the real number $\beta$ if there is some $M$ such that for all multiples $P$ of $L M$ there exists a $Q_{P}$ set $F$ with $\mu\left[S_{L}\left(Q_{P}\right) F\right] \geqslant \beta$. Let $\alpha$ be the supremum of the numbers $\beta$ for which some integer works. We will show that $\alpha=1$. This will prove the theorem, since for a finite subset $A$ of $G$, we have $S_{L}\left(Q_{P}\right) \subset \cap_{A} Q_{P}$ for sufficiently large $P$.

We first show that 4 works for $4^{-d}$, so that $\alpha>0$. Aperiodicity of the action guarantees that for every integer $P$ and every subset $E$ of positive measure, there is a $Q_{P}$ set of positive measure contained in $E$. Zorn's Lemma provides a maximal $Q_{P}$ set $F$; that is, a measurable subset $F$ such that if $F^{\prime} \supset F$ and $F^{\prime}$ is also a $Q_{P}$ set, then $\mu\left(F^{\prime} \backslash F\right)=0$. We claim that $\mu\left(R_{P} F\right)$ $=1$. For otherwise, $X \backslash R_{P} F$ would contain a $Q_{P}$ set of positive measure, and this could clearly be combined with $F$ to produce a larger $Q_{P}$ set. Now assume that $P$ is divisible by 4 . Then $R_{P}$ is covered by $4^{d}$ translates of $Q_{P / 2}$, and so there must be at least one such translate, say $Q_{P / 2}+t$, for which $\mu\left[\left(Q_{P / 2}+t\right) F\right] \geqslant 4^{-d}$. Choose $t^{\prime}$ so that $Q_{P / 2}+t-t^{\prime}=S_{4}\left(Q_{P}\right)$. Set $F_{1}$ $=t^{\prime} F$. Then $F_{1}$ is a $Q_{P}$ set and $\mu\left[S_{4}\left(Q_{P}\right) F_{1}\right]=\mu\left[\left(Q_{P / 2}+t\right) F\right] \geqslant 4^{-d}$.

Now suppose that $\beta<\alpha$. Let $\xi, \eta$ be positive numbers. We will show that $\beta-\xi+4^{-d}(1-\beta-\eta)$ is also less than $\alpha$. Hence $\beta+4^{-d}(1-\beta) \leqslant \alpha$. But the only number $\alpha$ in $(0,1]$ which can have this property for all $\beta<\alpha$ is $\alpha=1$.

The proof of our assertion is based on the following two statements.

(i) If $\eta>0$, then for every sufficiently large even integer $M$, integer $L$, multiple $P=N L M$ of $L M$, and $Q_{P}$ set $F$, for over $9 / 10$ of the elements $t$ in $Q_{P / L}$ we have

$$
\mu\left[B_{2 N}\left(Q_{P}\right)(t F)\right]<\eta .
$$

(ii) If $\xi>0$, then for every sufficiently large integer $L$, integer $M$, multiple $P=N L M$ of $L M$ by a multiple $N$ of $L$, and $Q_{P}$ set $F$, for over $9 / 10$ of the $t$ in $Q_{P / L}$ we have

$$
\mu\left[S_{L}\left(Q_{N}\right)\left(N Q_{P / N}\right)(t F)\right]>\mu\left[Q_{P}(t F)\right]-\xi .
$$

We will prove statement (i). The proof of (ii) is similar, and we omit it.

Divide $B_{2 N}\left(Q_{P}\right)$ into lower dimensional "slabs" as follows. For each nonempty subset $\Lambda$ of $\{1,2, \ldots, d\}$ and each $\sigma: \Lambda \rightarrow\{-1,1\}$, let

$$
\begin{aligned}
B_{\Lambda}^{\sigma}=\left\{t: 0 \leqslant t_{j}<P\right. & \text { if } j \notin \Lambda,-2 N \leqslant t_{j}<0 \text { if } \sigma(j)=-1, \\
P & \left.\leqslant t_{j}<P+2 N \text { if } \sigma(j)=1\right\} .
\end{aligned}
$$

Then $B_{2 N}\left(Q_{P}\right)$ is the disjoint union of the $B_{\Lambda}^{o}$. The number of these slabs is easily seen to be $3^{d}-1$. Let

$$
Q_{\Lambda}=\left\{2 N t: t_{j}=0 \text { if } j \notin \Lambda, 0 \leqslant t_{j}<M / 2 \text { if } j \in \Lambda\right\} .
$$

Since $B_{\Lambda}^{o}+Q_{\Lambda}$ is contained in a translate of $Q_{P}$, and since the collection $\left\{B_{\Lambda}^{\sigma}+t: t \in Q_{\Lambda}\right\}$ is disjoint, it follows that $B_{\Lambda}^{\sigma} F$ is a $Q_{\Lambda}$ set.

Let the cardinality of a set $A$ be denoted by $|A|$. Then $Q_{P / L}$ consists of a disjoint union of $\left|Q_{P / L}\right| /\left|Q_{\Lambda}\right|$ translates of $Q_{\Lambda}$. Thus 


$$
\sum_{t \in Q_{P / L}} \mu\left[B_{\Lambda}^{o}(t F)\right] \leqslant \frac{\left|Q_{P / L}\right|}{\left|Q_{\Lambda}\right|} .
$$

Since $\left|Q_{\Lambda}\right| \geqslant M / 2$, the right-hand side is bounded by $2\left|Q_{P / L}\right| / M$. Summing over $\Lambda$ and $\sigma$ shows that

$$
\sum_{t \in Q_{P / L}} \mu\left[B_{2 N}\left(Q_{P}\right)(t F)\right] \leqslant \frac{2\left(3^{d}-1\right)\left|Q_{P / L}\right|}{M},
$$

and hence that

$$
\frac{1}{\left|Q_{P / L}\right|}\left|\left\{t \in Q_{P / L}: \mu\left[B_{2 N}\left(Q_{P}\right)(t F)\right]>\frac{20\left(3^{d}-1\right)}{M}\right\}\right|<\frac{1}{10} .
$$

If $M>20\left(3^{d}-1\right) / \eta$, the desired inequality in (i) holds.

We now complete the proof of the theorem using (i) and (ii). Suppose $\beta<\alpha$, and choose $L_{1}$ to work for $\beta$. This means that there exists an $M_{1}$ such that for any multiple $P$ of $L_{1} M_{1}$ there is a $Q_{P}$ set $F$ with $\mu\left[S_{L_{1}}\left(Q_{P}\right) F\right] \geqslant \beta$. Let $M$ be an even multiple of $M_{1}$ and so large that (i) holds. Let $L$ be a multiple of $L_{1}$ and so large that (ii) holds. Let $P$ be a multiple of $L M$ by a multiple $N$ of $2 L$. Hence for the $Q_{P}$ set $F$ with $\mu\left[S_{L_{1}}\left(Q_{P}\right) F\right] \geqslant \beta$, there exists some $t$ in $Q_{P / L}$ so that the inequalities in both (i) and (ii) hold. Let $F_{1}=t F$, and let

$$
E=X \backslash\left(Q_{P} \cup B_{2 N}\left(Q_{P}\right)\right) F_{1} .
$$

Choose a maximal $Q_{N}$ set $F_{2}$ in $E$. Then, arguing as before, $E \subset R_{N} F_{2}$ except for a null set. Now $R_{N}$ is the union of $4^{d}$ translates of $Q_{N / 2}$, so for one of these, say $Q_{N / 2}+u$, we must have

$$
\mu\left[\left\{\left(Q_{N / 2}+u\right) F_{2}\right\} \cap E\right] \geqslant 4^{-d} \mu(E) .
$$

Choose $u^{\prime}$ so that $Q_{N / 2}+u-u^{\prime}=S_{4}\left(Q_{N}\right)$, and put $F_{3}=u^{\prime} F_{2}$. Finally, put $F_{4}=\left(N Q_{P / N}\right) F_{1}$. We will check that $F^{\prime}=F_{3} \cup F_{4}$ is a $Q_{N}$ set for which

$$
\mu\left[S_{L}\left(Q_{N}\right) F^{\prime}\right]>\beta-\xi+4^{-d}(1-\beta-\eta) .
$$

This will show that $L$ works for $\beta-\xi+4^{-d}(1-\beta-\eta)$, and complete the proof.

The set $F_{2}$ was chosen to be a $Q_{N}$ set, so the same holds for $F_{3}$. Since $F_{1}$ is a $Q_{P}$ set, it follows that $F_{4}$ is a $Q_{N}$ set, and

$$
Q_{N} F_{4}=\left(Q_{N}+N Q_{P / N}\right) F_{1}=Q_{P} F_{1} .
$$

Now $u^{\prime} \in R_{N}$, so that

$$
Q_{N} F_{3}=Q_{N}\left(u^{\prime} F_{2}\right) \subset R_{2 N} E .
$$

Since $R_{2 N} E$ is disjoint from $Q_{P} F_{1}$, we have that $F_{3} \cup F_{4}$ is also a $Q_{N}$ set.

We estimate the measures of the disjoint sets $S_{L}\left(Q_{N}\right) F_{3}$ and $S_{L}\left(Q_{N}\right) F_{4}$ separately. By (i) we have

$$
\begin{aligned}
\mu\left[S_{L}\left(Q_{N}\right) F_{3}\right] & \geqslant \mu\left[S_{4}\left(Q_{N}\right) F_{3}\right]=\mu\left[\left(Q_{N / 2}+u\right) F_{2}\right] \geqslant 4^{-d} \mu(E) \\
& \geqslant 4^{-d}\left(1-\mu\left[Q_{P}(t F)\right]-\mu\left[B_{2 N}\left(Q_{P}\right)(t F)\right]\right) \\
& \geqslant 4^{-d}\left(1-\mu\left[Q_{P}(t F)\right]-\eta\right) .
\end{aligned}
$$


Also, using (ii) we have

$$
\mu\left[S_{L}\left(Q_{N}\right) F_{4}\right]=\mu\left[S_{L}\left(Q_{N}\right)\left(N Q_{P / N}\right)(t F)\right] \geqslant \mu\left[Q_{P}(t F)\right]-\xi .
$$

Thus

$$
\mu\left[S_{L}\left(Q_{N}\right) F^{\prime}\right] \geqslant \mu\left[Q_{P}(t F)\right]-\xi+4^{-d}\left(1-\mu\left[Q_{P}(t F)\right]-\eta\right) .
$$

Since $t \in Q_{P / L}$, we have $Q_{P}+t \supset S_{L}\left(Q_{P}\right)$, so that

$$
\mu\left[Q_{P}(t F)\right] \geqslant \mu\left[S_{L}\left(Q_{P}\right) F\right] \geqslant \mu\left[S_{L_{1}}\left(Q_{P}\right) F\right] \geqslant \beta .
$$

Applying this to the right side of the previous inequality gives the desired result.

3. Hyperfiniteness. A nonsingular action of a countable group $G$ on $X$ is called hyperfinite if for each finite subset $A$ of $G$ and each $\varepsilon>0$, there exists some finite group $K \subset \Re(\mu)$ such that $K x \subset G x$ for almost every $x$, and such that for each $a \in A$ there is some $k \in K$ with $\mu(\{x: a x \neq k x\})<\varepsilon$.

This definition (in the measure-preserving case) is due to Dye [2]. Two equivalent definitions are the following:

(1) there is some nonsingular action of $\mathbf{Z}$ on $X$ such that $\mathbf{Z} x=G x$ for almost every $x$;

(2) there exist finite groups $G_{1} \subset G_{2} \subset \cdots$ of nonsingular transformations of $X$ with $\cup G_{n} x=G x$ for almost every $x$.

The proof of the equivalence of these with the original definition is in [2] and [5].

The first lemma describes the aperiodic decomposition of $X$.

LEMMA 1. Let the countable abelian group $G$ act nonsingularly on $X$. If $H$ is a subgroup of $G$, let $X_{H}=\{x: g x=x$ if and only if $g \in H\}$. Then $X$ is the disjoint union of the $X_{H}$, each $X_{H}$ is measurable and invariant under $G$, and $G / H$ acts aperiodically on $X_{H}$.

Proof. Clear.

LEMMA 2. A nonsingular action of $G$ is hyperfinite if for each finite subset $A$ of $G$ and each $\varepsilon>0$, there exists a finite subset $B$ of $G$ and $a B$ set $F$ in $X$ such that $\mu\left[\left(\cap_{A} B\right) F\right]>1-\varepsilon$.

Proof. Suppose that $A$ is a finite subset of $G$ which contains the identity, and let $\varepsilon>0$. Choose $B$ and $F$ to satisfy the hypothesis. We construct the required finite group $K$ from $B$ and $F$ as follows. For each permutation $\pi$ of $B$, let $T_{\pi} \in \mathcal{X}(\mu)$ be defined by

$$
T_{\pi} x= \begin{cases}\pi(b) x & \text { if } x \in b F(b \in B), \\ x & \text { if } x \in X \backslash B F .\end{cases}
$$

The collection of such $T_{\pi}$ forms a finite group $K$ in $\Re(\mu)$. Clearly $K x \subset G x$ for every $x$. If $a \in A$, the map $b \mapsto b+a$ from $\cap_{A} B$ to $B$ extends to a permutation $\pi_{a}$ of $B$. Then $T_{\pi_{a}} \in K$, and

$$
\mu\left(\left\{x: a x \neq T_{\pi_{a}} x\right\}\right) \geqslant \mu\left[\left(\bigcap_{A} B\right) F\right]>1-\varepsilon .
$$

THEOREM 2. Every nonsingular action of a countable abelian group on a Lebesgue space is hyperfinite. 
Proof. From the definition of hyperfiniteness, it is clear that it suffices to consider the case where $G$ is finitely generated. Since the number of subgroups of a finitely generated abelian group is countable, by Lemma 1 it suffices to consider aperiodic actions. The result then follows from Theorem 1 and Lemma 2.

REMARK. Lemma 2 gives a criterion for hyperfiniteness of nonsingular actions of countable groups which are not necessarily abelian. Our results show that all countable abelian groups satisfy this criterion. In the measurepreserving case, the conditions on $B$ and $F$ can be replaced by $\mu(B F)>1-$ $\varepsilon$ and $\left|\cap_{A} B\right|>(1-\varepsilon)|B|$, the latter being a condition only on the group and not the action.

\section{REFERENCES}

1. J. P. Conze, Entropie d'un groupe abélian de transformations, Z. Wahrscheinlichkeitstheorie und Verw. Gebiete 25(1972), 11-30.

2. H. A. Dye, On groups of measure preserving transformations. I, Amer. J. Math. 81 (1959), 119-159. MR 24 \# A1366.

3. __ On groups of measure preserving transformations. II, Amer. J. Math. 85(1963), 551-576. MR 28 \# 1275.

4. Y. Katznelson and B. Weiss, Commuting measure-preserving transformations, Israel J. Math. 12(1972), 161-173. MR 47 \# 5227.

5. W. Krieger, On nonsingular transformations of a measure space. I, Z. Wahrscheinlichkeitstheorie und Verw. Gebiete 11(1969), 83-97. MR 39 \#1628.

6. On the entropy of groups of measure preserving transformations (to appear).

7. D. A. Lind, Locally compact measure preserving flows, Advances in Math. (to appear).

8. V. A. Rohlin, On the fundamental ideas of measure theory, Mat. Sb. 25(67)(1949), 107-150; English transl., Amer. Math. Soc. Transl. (1)10(1962), 1-54. MR 11, 18.

9. S. Sakai, $C^{*}$-algebras and $W^{*}$-algebras, Springer-Verlag, New York, 1971.

10. A. M. Veršik, Nonmeasurable decompositions, orbit theory, algebras of operators, Dokl. Akad. Nauk SSSR 199(1971), 1004-1007 = Soviet Math. Dokl. 12(1971), 1218-1222. MR 44 \#453.

11. Appendix to Russian translation of Invariant means on topological groups and their applications, by F. P. Greenleaf.

Department of Mathematics, University of California, Berkeley, California 94720 